Research Article

\title{
Pressure of Urbanisation on the Fish Community Structure in Küçük Menderes River Basin (Turkey)
}

\section{Kentleşmenin Küçük Menderes Nehir Havzası (Türkiye) Balık Toplulukları Üzerindeki Baskısı}

\author{
Gülşah Saç*, Özcan Gaygusuz, Zeynep Dorak, Latife Köker Demo, Fatih Aydın, \\ Reyhan Akçaalan Albay, Meriç Albay \\ Istanbul University, Faculty of Aquatic Sciences, Department of Marine and Freshwater Resources \\ Management, 34134, Laleli, Istanbul, Turkey. \\ gulsahsac@gmail.com (https://orcid.org/0000-0001-9988-1116),ozcan.gaygusuz@istanbul.edu.tr \\ (https://orcid. org/0000-0001-6861-6221), zdorak@istanbul.edu.tr (https://orcid.org/0000-0003- \\ 4782-3082), latife.koker@istanbul.edu.tr (https://orcid.org/0000-0002-9134-2801), \\ fatih.aydin@istanbul.edu.tr (https://orcid.org/0000-0002-5003-2620), akcaalan@istanbul.edu.tr \\ (https://orcid.org/0000-0002-0756-8972), merbay@istanbul.edu.tr (https://orcid.org/0000-0001- \\ 9726-945X) \\ Received Date: 06.07.2020, Accepted Date: 23.12.2020
}

\begin{abstract}
Urbanization is linked to changes in aquatic flora and fauna, including fishes, and it often leads to a decline in fish diversity, richness, and density. This study aims to determine the fish fauna and their abundance in Küçük Menderes River Basin, which is under the threat of urbanization. The field surveys were carried out in 50 different locations in lotic and lentic biotopes, and fish sampling was performed by using electro-shocker, seine-net, and gillnets in November 2017 and May 2018. A total of 21 taxa belonging to 14 families (Anguillidae, Acheilognathidae, Atherinidae, Blenniidae, Cobitidae, Cyprinidae, Gobiidae, Leuciscidae, Mugilidae, Nemacheilidae, Percidae, Poecilidae, Siluridae and Syngnathidae) was identified. Cyprinidae has the highest species richness with four taxa and the most commonly found fish species were Squalius kosswigi and Cyprinus carpio. In terms of abundance of species, Gambusia holbrooki was the most dominant fish species in Hirsiz (the catch per unit effort $=23.28 \mathrm{n} / \mathrm{min} / \mathrm{m}^{2}$ ) and Kuskudan (the catch per unit effort $=24.00 \mathrm{n} / \mathrm{min} / \mathrm{m}^{2}$ ) streams. While Alburnus demiri, Barbus pergamonensis, Cobitis fahireae, Oxynoemacheilus germencicus, Petroleuciscus smyrnaeus and S. kosswigi are Anatolian endemics, Atherina boyeri, Carassius auratus, C. gibelio and Gambusia holbrooki are the invasive fish species in the basin.
\end{abstract}

Keywords: Water pollution, invasive fish, drought, endemic, irrigation

\section{Öz}

Kentleşme, balıkları da kapsayan sucul fauna ve floradaki değişimlerle bağlantılı olup sıklıkla balık çeşitliliği, zenginliği ve yoğunluğunda azalmaya yol açmaktadır. Bu çalışmanın amacı, şehirleşme tehdidi altındaki Küçük Menderes Nehir Havzası'nın balık faunası ile bunların bolluklarını belirlemektir. Saha çalışmaları, durgun ve akışlı biyotoplardaki 50 farklı istasyonda sürdürülmüş ve balık örneklemesi elektroşoker, ığrıp ve galsama ağları kullanılarak Kasım 2017 ve Mayıs 2018

\footnotetext{
* Corresponding author
} 
tarihlerinde gerçekleştirilmiştir. 14 familyaya (Anguillidae, Acheilognathidae, Atherinidae, Blenniidae, Cobitidae, Cyprinidae, Gobiidae, Leuciscidae, Mugilidae, Nemacheilidae, Percidae, Poecilidae, Siluridae ve Syngnathidae) ait toplam 21 takson tanımlanmıştır. Cyprinidae dört takson ile en yüksek tür çeşitliliğine sahiptir ve Squalius kosswigi ve Cyprinus carpio en yaygın balık türleri olarak tespit edilmiştir. Türler bollukları açısından değerlendirildiğinde, Gambusia holbrooki Hırsız (birim çaba başına düşen av miktarı $=23.28 \mathrm{adet} / \mathrm{dk} / \mathrm{m}^{2}$ ) ve Kuskudan (birim çaba başına düşen av miktarı $=24.00$ adet $/ \mathrm{dk} / \mathrm{m}^{2}$ ) derelerindeki en baskın balık türüdür. Alburnus demiri, Barbus pergamonensis, Cobitis fahireae, Oxynoemacheilus germencicus, Petroleuciscus smyrnaeus ve $S$. kosswigi havzadaki Anadolu endemikleri iken Atherina boyeri, Carassius auratus, C. gibelio ve Gambusia holbrooki istilacı balık türleridir.

Anahtar kelimeler: Su kirliliği, istilacı balıklar, kuraklık, endemik, sulama

\section{Introduction}

River basins have an essential role in the biosphere as a conduit for water and are used by humans for transportation, fisheries, hydropower, and domestic, industrial, and agricultural water supplies (Pompeu \& Alves, 2005). Urbanization is a process of the increasing number of people in an area and it leads to a horizontal or vertical growth of urban areas (Hassan Rashid et al., 2018). Both increased human population and growth in urban areas increase the pressure, such as pollution and rising water requirement for domestic use, on water resources. Therefore, urbanization is one of the major threats affecting the river basins (Shukla et al., 2013), and it is linked to changes in aquatic fauna, including fishes and it often leads to declines in fish diversity, richness, and density.

Küçük Menderes River, located in the centre of the west coastline of the Anatolia, flows through the province of İzmir. Today, İzmir is one of the biggest metropolitans of Turkey and it has been an important settlement area of the Western Anatolia since the ancient ages. According to "the level of urbanisation" that means the proportion of the population living in urban areas to the country's total population, İzmir is the third largest city of Turkey. The urbanization level of the city has increased rapidly by immigration with the urbanization models originating from trade, industry, tourism and agriculture. The boundaries of the urban areas have been expanded, especially with the administration of "metropolitan" status in 1984 and the urban population of Izmir, which was 818,251 in 1980, reached 1,489,772 in 1985 and 4,367,251 today (Işık, 2005; Turkey Statistical Institute [TURKSTAT], 2020). Due to the rapidly increasing population and urbanization, many aquatic and terrestrial ecosystems in the region are under threat (Kurucu \& Chiristina, 2008; Sökmen Y1lmaz \& Y1lmaz, 2019)

Küçük Menderes River receives its waters from the small streams in Kiraz lowland and it flows into the Aegean Sea in the west of Selçuk. There are three 
natural lakes (Belevi, Çatal and Gebekirse) and also some dam lakes built to supply drinking and irrigation water in the river basin. Küçük Menderes River has formed saline wetlands before reaching into the Aegean Sea and these wetlands were entitled as Selçuk Bird Paradise. In 1991 it was announced as $1^{\text {st }}$ and $2^{\text {nd }}$ Degree natural protected area by the Ministry of Culture and Tourism and in 1994 as wildlife protection area by the Ministry of Agriculture and Forestry (Sütgibi, 2009).

Nowadays, Küçük Menderes River is under the pressure of urbanization due to its fertile agricultural lands and high industrial and tourism potential. However, the lack of infrastructure (sewage, treatment plants, etc.) and rapid population growth in the basin cause water pollution/lose and damage water balance (Yildiz et al., 2009). Many of the streams have been turned to reservoirs to provide water for domestic, agriculture and industrial purposes for the metropolitan İzmir.

A limited number of studies were conducted about the distributions of the fishes in the streams and lakes of the river basin in the past (Karaman, 1972; Balik, 1979; Balık et al., 1995; Sarsu, 1981; İlhan et al., 2009). Karaman (1972) has described Squalius kosswigii as a new species from the Gümüldür Stream, which was located in the Tahtal Dam sub-basin before the construction of the dam. In the study on taxonomy and ecological characteristics of freshwater fishes in Western Anatolia, Balık (1979) has reported that eight fish species, Salaria fluviatilis (Asso y del Rio, 1801), Oxynoemacheilus germencicus (Erk'akan, Nalbant \& Özeren, 2007), Alburnus demiri Özuluğ \& Freyhof, 2008, Chondrostoma holmwoodii (Boulenger, 1896), Rhodeus amarus (Bloch, 1782), Alburnoides bipunctatus (Bloch, 1782) and S. kosswigii have been living in Gümüldür, Şaşal and Bulgurca streams. According to Sarsu (1981), freshwater fish species living in Gümüldür Stream and its associated sources have been reported as Anguilla anguilla (Linnaeus, 1758), Barbus pergamonensis Karaman, 1971, Cyprinus carpio Linnaeus, 1758, Cobitis fahireae Erk'akan, Atalay-Ekmekçi \& Nalbant, 1998, Mugil cephalus Linnaeus, 1758, Chelon aurata (Risso, 1810), A. demiri, C. holmwoodii, R. amarus, A. bipunctatus, $S$. kosswigi, O. germencicus and $S$. fluviatilis. As a faunistic study, Balik et al. (1995) have contributed to the fish fauna of the Tahtali Dam basin with three new species, Petroleuciscus smyrnaeus (Boulenger, 1896), Gambusia holbrooki Girard, 1859 and Chelon ramada (Risso, 1827). After the construction of the Tahtal1 Dam, İlhan et al. (2009) have studied some biological characteristics of Perca fluviatilis Linnaeus, 1758, which was introduced for stocking purposes to the dam. In the study about the length-weight relationships for freshwater fish species living in Küçük Menderes River Basin, Korkmaz et al. (2015) have reported the presence of Carassius gibelio (Bloch, 1782) and Carassius carassius (Linnaeus, 1758). 
These studies listed above have shown that there was no comprehensive study for the whole basin and only the fish species lists or some biological characteristics of these fishes in some locations of the basin were given and the abundance values of these fish species were not studied. The conservation and sustainability of biological diversity could be ensured by monitoring diversity using scientific methods. Therefore, it is very important to repeat the inventory and monitoring studies of fish fauna in the inland waters with time intervals (Özuluğ \& Saç, 2019). However, the presenting of abundance values of the existing fish species would be highly useful to reveal time-dependent changes for future studies. The main objective of this study is to determine the recent fish fauna living in Küçük Menderes River Basin. Additionally, by calculating the abundance values of these fish, it is likely to evaluate the pressures of urbanization.

\section{Method}

The field surveys were conducted in a total of 50 sampling sites (42 streams, 1 transitional water, 2 natural lakes and 5 dam lakes) in Küçük Menderes River Basin in November 2017 and May 2018 (Figure 1, Table 1).

\section{Figure 1}

\section{Sampling Sites in Küçük Menderes River Basin}

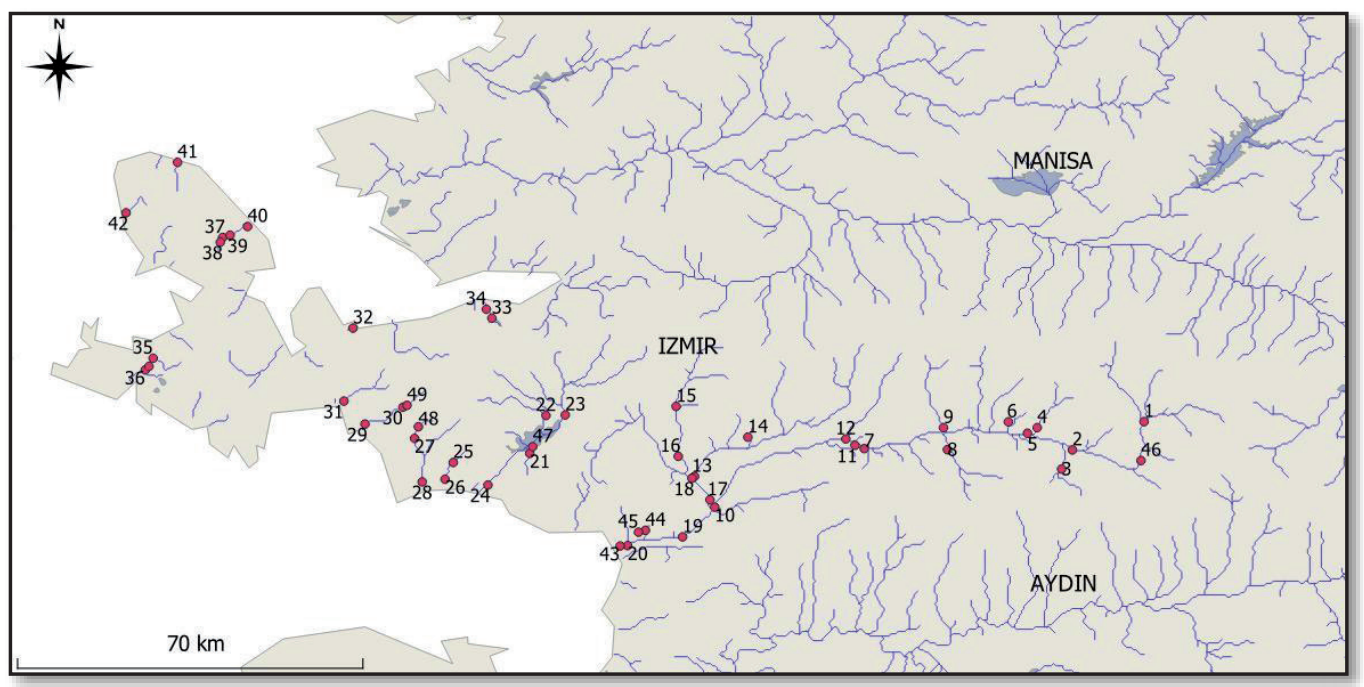


Gülşah Saç, Özcan Gaygusuz, Zeynep Dorak, Latife Köker Demo, Fatih Aydın, Reyhan Akçaalan Albay, Meriç Albay / Turkish Journal of Water Science \& Management 5 (1) (2021) / 40 - 58

\section{Table 1}

\section{Sampling Sites in Küçük Menderes River Basin with Coordinates}

\begin{tabular}{|c|c|c|c|}
\hline St. No & St. Name & Coordinates & Locality \\
\hline $1 *$ & Keleş Creek & $38.188261^{\circ} \mathrm{N}, 28.225178^{\circ} \mathrm{E}$ & Kiraz, İzmir \\
\hline $2 *$ & Taşavra Creek & $38.136694^{\circ} \mathrm{N}, 28.094658^{\circ} \mathrm{E}$ & Beydağ, İzmir \\
\hline $3 *$ & Pirinççi Creek & $38.102115^{\circ} \mathrm{N}, 28.074048^{\circ} \mathrm{E}$ & Ödemiş, İzmir \\
\hline $4 * *$ & Gelinbözü Stream & $38.177378^{\circ} \mathrm{N}, 28.030595^{\circ} \mathrm{E}$ & Ödemiş, İzmir \\
\hline $5 * *$ & Küçük Menderes River-1 & $38.165631^{\circ} \mathrm{N}, 28.013342^{\circ} \mathrm{E}$ & Ödemiş, İzmir \\
\hline $6 * *$ & Zeytinlik Creek & $38.187889^{\circ} \mathrm{N}, 27.978032^{\circ} \mathrm{E}$ & Ödemiş, İzmir \\
\hline $7 * * *$ & Rahmanlar Stream & $38.140291^{\circ} \mathrm{N}, 27.715971^{\circ} \mathrm{E}$ & Tire, İzmir \\
\hline 8 & Eğridere Stream & $38.137058^{\circ} \mathrm{N}, 27.866841^{\circ} \mathrm{E}$ & Tire, İzmir \\
\hline $9 *$ & Aktaş Creek & $38.177333^{\circ} \mathrm{N}, 27.859872^{\circ} \mathrm{E}$ & Ödemiş, İzmir \\
\hline $10 *$ & Küçük Menderes River-2 & $38.032566^{\circ} \mathrm{N}, 27.443286^{\circ} \mathrm{E}$ & Torbalı, İzmir \\
\hline $11 * * *$ & Küçük Menderes River-3 & $38.145197^{\circ} \mathrm{N}, 27.698732^{\circ} \mathrm{E}$ & Tire, İzmir \\
\hline $12 * *$ & Falaka Creek & $38.156692^{\circ} \mathrm{N}, 27.682031^{\circ} \mathrm{E}$ & Bayındır, İzmir \\
\hline $13 * *$ & Ilica Stream & $38.088649^{\circ} \mathrm{N}, 27.406979^{\circ} \mathrm{E}$ & Torbal1, İzmir \\
\hline $14 * *$ & Uladi Creek & $38.160030^{\circ} \mathrm{N}, 27.503622^{\circ} \mathrm{E}$ & Bayındır, İzmir \\
\hline $15^{*}$ & Fetrek Creek-1 & $38.215895^{\circ} \mathrm{N}, 27.373613^{\circ} \mathrm{E}$ & Torbalı, İzmir \\
\hline $16 * * *$ & Fetrek Creek-2 & $38.124985^{\circ} \mathrm{N}, 27.378049^{\circ} \mathrm{E}$ & Torbalı, İzmir \\
\hline $17 * * *$ & Çevlik Creek-1 & $38.045394^{\circ} \mathrm{N}, 27.435825^{\circ} \mathrm{E}$ & Torbalı, İzmir \\
\hline $18 * * *$ & Çevlik Creek-2 & $38.085461^{\circ} \mathrm{N}, 27.402874^{\circ} \mathrm{E}$ & Torbalı, İzmir \\
\hline $19 * * *$ & Küçük Menderes River-4 & $37.978428^{\circ} \mathrm{N}, 27.384794^{\circ} \mathrm{E}$ & Selçuk, İzmir \\
\hline $20 * * *$ & Küçük Menderes River-5 & $37.963007^{\circ} \mathrm{N}, 27.283960^{\circ} \mathrm{E}$ & Selçuk, İzmir \\
\hline $21 *$ & Değirmendere Stream & $38.130352^{\circ} \mathrm{N}, 27.106610^{\circ} \mathrm{E}$ & Menderes, İzmir \\
\hline 22 & Şaşal Stream & $38.199433^{\circ} \mathrm{N}, 27.136033^{\circ} \mathrm{E}$ & Menderes, İzmir \\
\hline 23 & Tahtalı Creek & $38.200436^{\circ} \mathrm{N}, 27.171187^{\circ} \mathrm{E}$ & Menderes, İzmir \\
\hline $24 * *$ & Koca Creek & $38.072829^{\circ} \mathrm{N}, 27.030365^{\circ} \mathrm{E}$ & Menderes, İzmir \\
\hline $25^{*}$ & Ürkmez Stream-1 & $38.114544^{\circ} \mathrm{N}, 26.966368^{\circ} \mathrm{E}$ & Seferihisar, İzmir \\
\hline $26 * *$ & Ürkmez Stream-2 & $38.083291^{\circ} \mathrm{N}, 26.952555^{\circ} \mathrm{E}$ & Seferihisar, İzmir \\
\hline $27 * *$ & Karakoç Stream-1 & $38.157961^{\circ} \mathrm{N}, 26.898151^{\circ} \mathrm{E}$ & Seferihisar, İzmir \\
\hline 28 & Karakoç Stream-2 & $38.083766^{\circ} \mathrm{N}, 26.909176^{\circ} \mathrm{E}$ & Seferihisar, İzmir \\
\hline $29 * *$ & Yassidere Stream-1 & $38.184390^{\circ} \mathrm{N}, 26.806520^{\circ} \mathrm{E}$ & Seferihisar, İzmir \\
\hline $30 * *$ & Yassidere Stream-2 & $38.214620^{\circ} \mathrm{N}, 26.875686^{\circ} \mathrm{E}$ & Seferihisar, İzmir \\
\hline 31 & Azmak Stream & $38.225769^{\circ} \mathrm{N}, 26.767904^{\circ} \mathrm{E}$ & Seferihisar, İzmir \\
\hline $32 * *$ & Akpinar Stream & $38.357671^{\circ} \mathrm{N}, 26.784006^{\circ} \mathrm{E}$ & Urla, İzmir \\
\hline $33 *$ & Kona Stream-1 & $38.384826^{\circ} \mathrm{N}, 27.033001^{\circ} \mathrm{E}$ & Balçova, İzmir \\
\hline $34 * *$ & Kona Stream- 2 & $38.392633^{\circ} \mathrm{N}, 27.028781^{\circ} \mathrm{E}$ & Balçova, İzmir \\
\hline $35 * *$ & İncirli Stream & $38.303509^{\circ} \mathrm{N}, 26.420841^{\circ} \mathrm{E}$ & Çeşme, İzmir \\
\hline 36 & Hirsiz Stream & $38.282206^{\circ} \mathrm{N}, 26.404382^{\circ} \mathrm{E}$ & Çeşme, İzmir \\
\hline $37 * *$ & K1sık Stream & $38.523252^{\circ} \mathrm{N}, 26.547126^{\circ} \mathrm{E}$ & Karaburun, İzmir \\
\hline $38 * *$ & Kışla Stream & $38.514904^{\circ} \mathrm{N}, 26.542971^{\circ} \mathrm{E}$ & Karaburun, İzmir \\
\hline $39 *$ & Kuskudan Stream-1 & $38.528542^{\circ} \mathrm{N}, 26.561015^{\circ} \mathrm{E}$ & Karaburun, İzmir \\
\hline $40 * *$ & Kuskudan Stream-2 & $38.543320^{\circ} \mathrm{N}, 26.592170^{\circ} \mathrm{E}$ & Karaburun, İzmir \\
\hline $41 * *$ & Uzun Stream & $38.660341^{\circ} \mathrm{N}, 26.465133^{\circ} \mathrm{E}$ & Karaburun, İzmir \\
\hline $42 * *$ & Sudeğirmeni Stream & $38.568424^{\circ} \mathrm{N}, 26.371368^{\circ} \mathrm{E}$ & Karaburun, İzmir \\
\hline 43 & Transitional water & $37.959942^{\circ} \mathrm{N}, 27.264050^{\circ} \mathrm{E}$ & Selçuk, İzmir \\
\hline 44 & Çatal Lake & $37.990383^{\circ} \mathrm{N}, 27.317102^{\circ} \mathrm{E}$ & Selçuk, İzmir \\
\hline 45 & Gebekirse Lake & $37.986933^{\circ} \mathrm{N}, 27.304530^{\circ} \mathrm{E}$ & Selçuk, İzmir \\
\hline 46 & Beydağ Dam Lake & $38.117427^{\circ} \mathrm{N}, 28.219555^{\circ} \mathrm{E}$ & Beydağ, İzmir \\
\hline 47 & Tahtalı Dam Lake & $38.142752^{\circ} \mathrm{N}, 27.111891^{\circ} \mathrm{E}$ & Menderes, İzmir \\
\hline 48 & Kavakdere Dam Lake & $38.178899^{\circ} \mathrm{N}, 26.903701^{\circ} \mathrm{E}$ & Seferihisar, İzmir \\
\hline 49 & Seferihisar Dam Lake & $38.217997^{\circ} \mathrm{N}, 26.882979^{\circ} \mathrm{E}$ & Seferihisar, İzmir \\
\hline 50 & Alaçatı Dam Lake & $38.288922^{\circ} \mathrm{N}, 26.413136^{\circ} \mathrm{E}$ & Çeşme, İzmir \\
\hline
\end{tabular}


In streams, fish samples were collected by electro-shocker (SAMUS 725-G portable electro-shockers; frequency $50-55 \mathrm{~Hz} ; 20-60 \mathrm{~cm}$ fishing depth) from the stations and sampling was conducted opposite direction of the water flow (European Committee for Standardization [CEN], 2003). In transitional waters, seine nets (5 $\mathrm{mm}$ mesh size, $30 \mathrm{~m}$ in length and $2 \mathrm{~m}$ in width) were used (CEN, 2006).

In lakes, fish samples were captured using benthic and pelagic multi-mesh gillnets. Benthic gillnets (30 $\mathrm{m}$ in length and $1.5 \mathrm{~m}$ in width) have 12 different mesh sizes from $5 \mathrm{~mm}$ to $55 \mathrm{~mm}$. Pelagic gillnets $(27.5 \mathrm{~m}$ in length and $6.0 \mathrm{~m}$ in width) have 11 different mesh sizes from $6.25 \mathrm{~mm}$ to $55 \mathrm{~mm}$ (CEN, 2015). Additionally, in shorelines of lakes, a scoop-net ( $2 \mathrm{~mm}$ mesh sized) was used to capture small fishes.

Collected specimens were sacrificed with a lethal solution of anaesthesia (clove oil) and then transferred to a 5\% formaldehyde solution. In the laboratory, for each individual, standard length (SL) was measured to the nearest $0.1 \mathrm{~cm}$, and total body weight $(\mathrm{W})$ was weighed on a digital balance with a $0.01 \mathrm{~g}$ accuracy.

To determine the fish abundance, the catch per unit effort (CPUE) was calculated using the following equation; $\mathrm{CPUE}=(\mathrm{n} / \mathrm{t} / \mathrm{a}) \times 100(\mathrm{n}$ : specimen numbers, t: time (minute ( $\mathrm{min}$ ) for streams and hour (h) for lakes, a: sampling area) (Jordan \& Willis, 2001; Mehner et al., 2005). In streams, the sampling area means the area sampled by electro-shocker and the fish samples were captured from a maximum $200 \mathrm{~m}$ length of sampling sections in a maximum of 20 minutes. In lakes, the sampling area means the gillnet area used for the fish catch and the maximum length of time the nets stayed in the lake was limited to 12-16 hours. For the fish species caught with seine-net and scoop-net, CPUE was not estimated.

\section{Results}

Fish were captured in 38\% (19 stations) of the 50 stations, however, there were no fish in other stations due to pollution (7 stations) and drought (24 stations). A total of 21 fish species belonging to 14 families were determined in the river basin (Table 2). Cyprinidae has the highest species richness with four taxa and the most commonly found fish species were Squalius kosswigi (8 stations) and Cyprinus carpio (7 stations).

In terms of abundance of fish species, CPUE $\left(\mathrm{n} / \mathrm{t} / \mathrm{m}^{2}\right)$ values ranged from 0.01 to 24.00 in stream stations and G. holbrooki was the most dominant fish species in the Hirsiz $\left(\right.$ CPUE $\left.=23.28 \mathrm{n} / \mathrm{min} / \mathrm{m}^{2}\right)$ and Kuskudan $\left(\right.$ CPUE $\left.=24.00 \mathrm{n} / \mathrm{min} / \mathrm{m}^{2}\right)$ streams. In lakes, CPUE $\left(\mathrm{n} / \mathrm{h} / \mathrm{m}^{2}\right)$ values ranged from 0.01 to 62.43 and $P$. fluviatilis 
was the most dominant fish species in Beydağ Dam Lake (CPUE $=62.43 \mathrm{n} / \mathrm{h} / \mathrm{m}^{2}$ ). Fish species with a CPUE $\left(\mathrm{n} / \mathrm{min} / \mathrm{m}^{2}\right.$ and $\left.\mathrm{n} / \mathrm{h} / \mathrm{m}^{2}\right)$ value of 1 and above are determined as $R$. amarus, $C$. carpio, A. demiri, P. smyrnaeus, S. kosswigi, Chelon sp., O. germencicus, P. fluviatilis and G. holbrooki. While A. demiri, B. pergamonensis, C. fahireae, O. germencicus, P. smyrnaeus and S. kosswigi are Anatolian endemics, A. boyeri (Kavakdere Dam Lake), C. auratus, C. gibelio and $G$. holbrooki are the invasive fish species in the basin (Table 3).

\section{Table 2}

Fish Species Captured from Küçük Menderes River Basin and the Distribution of These Fishes by Sites

\begin{tabular}{|c|c|}
\hline Fish species & Stations \\
\hline $\begin{array}{l}\text { Familia: Acheilognathidae } \\
\quad \text { Rhodeus amarus (Bloch, 1782) }\end{array}$ & 22 \\
\hline $\begin{array}{l}\text { Familia: Anguillidae } \\
\quad \text { Anguilla anguilla (Linnaeus, 1758) }\end{array}$ & 44 \\
\hline $\begin{array}{l}\text { Familia: Atherinidae } \\
\quad \text { Atherina boyeri } \text { Risso } 1810 \\
\end{array}$ & 45,48 \\
\hline $\begin{array}{l}\text { Familia: Blenniidae } \\
\quad \text { Salaria fluviatilis (Asso, 1801) } \\
\end{array}$ & 21 \\
\hline $\begin{array}{l}\text { Familia: Cobitidae } \\
\quad \text { Cobitis fahireae Erk'akan, Atalay-Ekmekçi \& Nalbant } 1998\end{array}$ & 23,44 \\
\hline $\begin{array}{l}\text { Familia: Cyprinidae } \\
\text { Barbus pergamonensis Karaman 1971 } \\
\text { Carassius auratus (Linnaeus, 1758) } \\
\text { Carassius gibelio (Bloch, 1782) } \\
\text { Cyprinus carpio Linnaeus, 1758 }\end{array}$ & $\begin{array}{l}15,22 \\
50 \\
15,23,44,46,47,50 \\
44,45,46,47,48,49,50\end{array}$ \\
\hline $\begin{array}{l}\text { Familia: Gobiidae } \\
\quad \text { Knipowitschia caucasica }(\text { Berg, 1916) } \\
\end{array}$ & 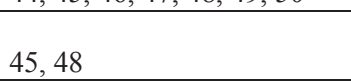 \\
\hline $\begin{array}{l}\text { Familia: Leuciscidae } \\
\text { Alburnus demiri Özuluğ \& Freyhof, } 2008 \\
\text { Petroleuciscus smyrnaeus (Boulenger, 1896) } \\
\text { Squalius kosswigi (Karaman, 1972) }\end{array}$ & $\begin{array}{l}22,23,45 \\
8,21,23,44,45,46 \\
15,22,23,25,28,31,33,48\end{array}$ \\
\hline $\begin{array}{l}\text { Familia: Mugilidae } \\
\text { Chelon aurata }(\text { Risso, } 1810) \\
\text { Chelon ramada }(\text { Risso, } 1827) \\
\text { Chelon } \mathrm{sp} .\end{array}$ & $\begin{array}{l}45 \\
45,43 \\
28,31\end{array}$ \\
\hline $\begin{array}{l}\text { Familia: Nemacheilidae } \\
\quad \text { Oxynoemacheilus germencicus (Erk'akan, Nalbant \& Özeren, 2007) }\end{array}$ & $15,22,23$ \\
\hline $\begin{array}{l}\text { Familia: Percidae } \\
\quad \text { Perca fluviatilis Linnaeus, } 1758 \\
\end{array}$ & $46,47,49$ \\
\hline $\begin{array}{l}\text { Familia: Poecilidae } \\
\quad \text { Gambusia holbrooki Girard, } 1859\end{array}$ & $36,39,44,45,48,50$ \\
\hline $\begin{array}{l}\text { Familia: Siluridae } \\
\quad \text { Silurus glanis Linnaeus, } 1758\end{array}$ & (1) \\
\hline $\begin{array}{l}\text { Familia: Syngnathidae } \\
\quad \text { Syngnathus abaster Risso, } 1827\end{array}$ & 45 \\
\hline
\end{tabular}


Gülşah Saç, Özcan Gaygusuz, Zeynep Dorak, Latife Köker Demo, Fatih Aydın, Reyhan Akçaalan Albay, Meriç Albay / Turkish Journal of Water Science \& Management 5 (1) (2021) / 40 - 58

\section{Table 3}

The Individual Numbers, Body Size and Weight Distributions and Sampling Sites of Fish Species Captured in Küçük Menderes River Basin

\begin{tabular}{|c|c|c|c|c|c|}
\hline Fish species & St. No & $\mathrm{n}$ & $\begin{array}{c}\mathrm{TL}(\mathrm{cm}) \\
\min -\max \end{array}$ & $\begin{array}{c}\text { W }(g) \\
\min -\max \end{array}$ & $\begin{array}{c}\text { CPUE } \\
\left(\mathrm{n} / \mathrm{min} / \mathrm{m}^{2} ;\right. \\
\left.\mathrm{n} / \mathrm{h} / \mathrm{m}^{2}\right)\end{array}$ \\
\hline Rhodeus amarus & $22^{\mathrm{S}, 1}$ & 29 & $2.6-5.3$ & $0.17-2.49$ & 1.66 \\
\hline Anguilla anguilla & $44^{\mathrm{A}}$ & 2 & $43.9-50.5$ & $148.24-191.03$ & $*$ \\
\hline \multirow[t]{2}{*}{ Atherina boyeri } & $45^{\mathrm{A}, 2}$ & 1 & 6.4 & 1.60 & 0.03 \\
\hline & $48^{\mathrm{A}, 2}$ & 1 & 7.8 & 3.08 & 0.02 \\
\hline Salaria fluviatilis & $21^{\mathrm{S}, 1}$ & 20 & $3.4-6.7$ & $0.40-3.50$ & 0.76 \\
\hline \multirow[t]{3}{*}{ Cobitis fahireae } & $23^{\mathrm{A}, 1}$ & 1 & 5.3 & 0.92 & 0.03 \\
\hline & $23^{\mathrm{S}, 1}$ & 7 & $5.1-6.8$ & $0.94-2.47$ & 0.06 \\
\hline & $44^{\mathrm{A}}$ & 1 & 5.3 & 0.84 & $*$ \\
\hline \multirow[t]{2}{*}{ Barbus pergamonensis } & $15^{\mathrm{S}, 1}$ & 1 & 6.3 & 3.62 & 0.05 \\
\hline & $22^{\mathrm{S}, 1}$ & 3 & $6.9-9.5$ & $4.36-12.26$ & 0.17 \\
\hline Carassius auratus & $50^{\mathrm{A}, 2}$ & 4 & $8.9-14.5$ & $12.07-45.56$ & 0.17 \\
\hline \multirow{9}{*}{ Carassius gibelio } & $15^{\mathrm{S}, 1}$ & 6 & $3.1-4.8$ & $0.57-2.14$ & 0.30 \\
\hline & $23^{\mathrm{S}, 1}$ & 1 & 31.7 & 743.49 & 0.01 \\
\hline & $44^{\mathrm{A}, 2}$ & 19 & $13.0-20.3$ & $39.41-144.52$ & 0.32 \\
\hline & $44^{\mathrm{S}, 2}$ & 22 & $16.7-23.7$ & $65.46-242.11$ & 0.64 \\
\hline & $46^{\mathrm{A}, 2}$ & 12 & $15.5-19.8$ & $70.72-153.05$ & 0.31 \\
\hline & $46^{\mathrm{S}, 2}$ & 6 & $17.8-32.0$ & $86.34-561.33$ & 0.10 \\
\hline & $47^{\mathrm{A}, 2}$ & 21 & $12.2-30.4$ & $27.18-631.71$ & 0.44 \\
\hline & $47^{\mathrm{S}, 2}$ & 15 & $20.0-33.3$ & $174.4-796.43$ & 0.20 \\
\hline & $50^{\mathrm{A}, 2}$ & 4 & $8.9-15.9$ & $9.27-47.79$ & 0.17 \\
\hline \multirow[t]{9}{*}{ Cyprinus carpio } & $44^{\mathrm{S}, 2}$ & 1 & 24.5 & 200.65 & 0.03 \\
\hline & $45^{\mathrm{A}, 2}$ & 1 & 33.0 & 532.15 & 0.47 \\
\hline & $46^{\mathrm{A}, 2}$ & 8 & $13.8-18.2$ & $39.74-87.84$ & 0.21 \\
\hline & $47^{\mathrm{A}, 2}$ & 1 & 22.6 & 160.08 & 0.02 \\
\hline & $48^{\mathrm{A}, 2}$ & 3 & $23.1-24.3$ & $234.29-255.38$ & 0.05 \\
\hline & $48^{\mathrm{S}, 2}$ & 1 & 27.7 & 386.22 & 0.02 \\
\hline & $49^{\mathrm{S}, 2}$ & 10 & $22.5-34.1$ & $156.36-734.19$ & 0.14 \\
\hline & $50^{\mathrm{A}, 2}$ & 209 & $7.0-21.8$ & $5.17-136.83$ & 8.93 \\
\hline & $50^{\mathrm{S}, 2}$ & 22 & $9.7-22.6$ & $13.51-169.44$ & 0.72 \\
\hline \multirow[t]{4}{*}{ Knipowitschia caucasica } & $45^{\mathrm{A}}$ & 1 & 2.3 & 0.1 & $*$ \\
\hline & $45^{\mathrm{S}}$ & 14 & $1.6-3.0$ & $0.04-0.26$ & $*$ \\
\hline & $48^{\mathrm{A}}$ & 1 & 3.2 & 0.35 & $*$ \\
\hline & $48^{\mathrm{S}}$ & 1 & 2.7 & 0.20 & $*$ \\
\hline \multirow[t]{4}{*}{ Alburnus demiri } & $22^{\mathrm{A}, 1}$ & 1 & 6.7 & 4.40 & 0.04 \\
\hline & $22^{\mathrm{S}, 1}$ & 20 & $5.9-8.5$ & $1.79-6.29$ & 1.14 \\
\hline & $23^{\mathrm{A}, 1}$ & 4 & $3.6-5.4$ & $0.32-0.95$ & 0.11 \\
\hline & $45^{\mathrm{A}, 2}$ & 4 & $14.8-15.8$ & $29.36-36.35$ & 0.12 \\
\hline \multirow[t]{6}{*}{ Petroleuciscus smyrnaeus } & $8^{S, 1}$ & 29 & $1.8-8.5$ & $0.06-8.50$ & 0.52 \\
\hline & $21^{\mathrm{S}, 1}$ & 69 & $2.9-8.2$ & $0.25-8.18$ & 2.63 \\
\hline & $23^{\mathrm{A}, 1}$ & 1 & 6.1 & 3.43 & 0.03 \\
\hline & $23^{\mathrm{S}, 1}$ & 1 & 7.4 & 8.13 & 0.01 \\
\hline & $44^{\mathrm{A}, 2}$ & 50 & $9.1-12.0$ & $10.14-23.10$ & 0.85 \\
\hline & $44^{\mathrm{S}, 2}$ & 9 & $9.0-10.4$ & $9.66-15.40$ & 0.26 \\
\hline
\end{tabular}

${ }^{\text {A: }}$ Autumn; ${ }^{\text {S: Spring; }}{ }^{1}$ :Electro-shocker; ${ }^{2}$ : Gillnet; ${ }^{3}$ : Beach seine-net; $*$ Scoop net; $* *$ Seine-net 
Gülşah Saç, Özcan Gaygusuz, Zeynep Dorak, Latife Köker Demo, Fatih Aydın, Reyhan Akçaalan Albay, Meriç Albay / Turkish Journal of Water Science \& Management 5 (1) (2021) / 40 - 58

Table 3

(Continued)

\begin{tabular}{|c|c|c|c|c|c|}
\hline Fish species & St. No & $\mathrm{n}$ & $\begin{array}{c}\text { TL }(\mathrm{cm}) \\
\min -\max \end{array}$ & $\begin{array}{c}\mathrm{W}(\mathrm{g}) \\
\min -\max \end{array}$ & $\begin{array}{c}\text { CPUE } \\
\left(\mathrm{n} / \mathrm{min} / \mathrm{m}^{2}\right. \\
\left.\mathrm{n} / \mathrm{h} / \mathrm{m}^{2}\right)\end{array}$ \\
\hline \multirow[t]{2}{*}{ P. smyrnaeus (continued) } & $45^{\mathrm{A}, 2}$ & 16 & $8.5-11.3$ & $8.93-44.81$ & 0.03 \\
\hline & $46^{\mathrm{A}, 2}$ & 2 & $9.3-10.4$ & $11.65-15.81$ & 0.05 \\
\hline \multirow[t]{11}{*}{ Squalius kosswigi } & $15^{\mathrm{S}, 1}$ & 4 & $2.4-3.7$ & $0.15-0.59$ & 0.20 \\
\hline & $22^{\mathrm{A}, 1}$ & 1 & 6.6 & 2.97 & 0.04 \\
\hline & $22^{\mathrm{S}, 1}$ & 37 & $3.2-11.8$ & $0.22-23.05$ & 2.11 \\
\hline & $23^{\mathrm{A}, 1}$ & 6 & $3.5-6.3$ & $0.47-2.92$ & 0.17 \\
\hline & $23^{\mathrm{S}, 1}$ & 25 & $1.8-13.8$ & $0.05-36.06$ & 0.22 \\
\hline & $25^{\mathrm{S}, 1}$ & 11 & $2.2-14.1$ & $0.07-36.57$ & 0.11 \\
\hline & $28^{\mathrm{S}, 1}$ & 4 & $2.4-2.9$ & $0.12-0.27$ & 0.04 \\
\hline & $31^{\mathrm{S}, 1}$ & 40 & $4.2-13.8$ & $0.73-35.68$ & 5.13 \\
\hline & $33^{\mathrm{S}, 1}$ & 60 & $4.3-15.3$ & $0.84-52.52$ & 3.00 \\
\hline & $48^{\mathrm{A}, 2}$ & 94 & $10.1-23.9$ & $11.69-183.65$ & 1.65 \\
\hline & $48^{\mathrm{S}, 2}$ & 17 & $11.6-17.5$ & $16.60-58.12$ & 0.33 \\
\hline \multirow[t]{2}{*}{ Chelon aurata } & $45^{\mathrm{A}, 2}$ & 21 & $15.5-36.0$ & $25.15-356.63$ & 0.61 \\
\hline & $45^{\mathrm{S}, 2}$ & 1 & 20.0 & 56.98 & 0.02 \\
\hline \multirow[t]{3}{*}{ Chelon ramada } & $43^{\mathrm{A}, 3}$ & 4 & $9.8-12.9$ & $8.5-16.12$ & $* *$ \\
\hline & $43^{\mathrm{S}, 3}$ & 2 & $13.2-24.5$ & $13.60-135.11$ & $* *$ \\
\hline & $45^{\mathrm{A}, 2}$ & 1 & 35.0 & 374.22 & 0.03 \\
\hline \multirow[t]{3}{*}{ Chelon sp. } & $28^{\mathrm{A}, 1}$ & 20 & $2.4-3.1$ & $0.12-0.33$ & 0.48 \\
\hline & $28^{\mathrm{S}, 1}$ & 1 & 2.7 & 0.20 & 0.01 \\
\hline & $31^{\mathrm{S}, 1}$ & 17 & $2.3-4.8$ & $0.10-1.07$ & 2.18 \\
\hline \multirow{4}{*}{$\begin{array}{l}\text { Oxynoemacheilus } \\
\text { germencicus }\end{array}$} & $15^{\mathrm{s}, 1}$ & 58 & $2.2-4.1$ & $0.08-0.79$ & 2.90 \\
\hline & $22^{\mathrm{S}, 1}$ & 34 & $2.6-4.2$ & $0.13-0.75$ & 1.94 \\
\hline & $23^{\mathrm{A}, 1}$ & 12 & $4.7-6.2$ & $0.89-2.31$ & 0.33 \\
\hline & $23^{\mathrm{S}, 1}$ & 1 & 6.5 & 2.50 & 0.01 \\
\hline \multirow[t]{6}{*}{ Perca fluviatilis } & $46^{\mathrm{A}, 2}$ & 32 & $6.2-25.0$ & $2.78-275.86$ & 0.82 \\
\hline & $46^{\mathrm{S}, 2}$ & 3933 & $1.1-29.8$ & $9.02-492.84$ & 62.43 \\
\hline & $47^{\mathrm{A}, 2}$ & 81 & $13.0-29.2$ & $27.22-375.65$ & 169.00 \\
\hline & $47^{\mathrm{S}, 2}$ & 115 & $14.2-30.4$ & $33.00-443.69$ & 1.52 \\
\hline & $49^{\mathrm{A}, 2}$ & 2 & $24.2-26.7$ & $193.34-290.76$ & 0.05 \\
\hline & $49^{\mathrm{S}, 2}$ & 44 & $15.4-36.1$ & $50.78-864.80$ & 0.62 \\
\hline \multirow[t]{8}{*}{ Gambusia holbrooki } & $36^{\mathrm{A}, 1}$ & 149 & $1.2-3.9$ & $0.03-0.48$ & 23.28 \\
\hline & $36^{\mathrm{S}, 1}$ & 60 & $1.4-4.3$ & $0.01-0.94$ & 10.00 \\
\hline & $39^{\mathrm{S}, 1}$ & 24 & $2.5-4.8$ & $0.15-1.32$ & 24.00 \\
\hline & $44^{\mathrm{A}}$ & 30 & $1.4-2.8$ & $0.03-0.19$ & $*$ \\
\hline & $44^{\mathrm{S}}$ & 21 & $1.6-3.0$ & $0.05-0.29$ & * \\
\hline & $45^{\mathrm{s}}$ & 20 & $1.7-4.8$ & $0.04-0.63$ & * \\
\hline & $48^{\mathrm{A}}$ & 24 & $1.8-3.6$ & $0.06-0.48$ & $*$ \\
\hline & $50^{\mathrm{A}}$ & 1 & 1.6 & 0.04 & * \\
\hline Silurus glanis & $47^{\mathrm{A}, 2}$ & 1 & 26.8 & 105.88 & 0.02 \\
\hline Syngnathus abaster & $45^{\mathrm{s}}$ & 3 & $6.0-7.2$ & $0.10-0.19$ & $*$ \\
\hline
\end{tabular}

A: Autumn; ${ }^{\text {S: Spring; }}{ }^{1}$ :Electro-shocker; ${ }^{2}$ : Gillnet; ${ }^{3}$ : Beach seine-net; *Scoop net; **Seine-net 


\section{Discussion and Conclusion}

As a result of the observations made in the field, remarkable four problems are determined: (1) dryness due to atmospheric conditions and/or overuse of water in domestic/industrial sector/agricultural irrigation, (2) water pollution, (3) the presence of invasive-alien or translocated fish and (4) deterioration of river continuity (horizontally and vertically) due to hydraulic structures such as dams, flood protection structures and culverts. Many of these problems affecting Küçük Menderes River basin are thought to be the consequences of the pressures of the increasing urbanization level. We observed that while drought is the main problem for some stations, two or more problems mentioned above are effective together in the others.

In river ecosystems, fish could not be caught in total 31 stations in both sampling periods, and also19 of them were dry in both seasons. Even though 5 of them were wet in one sampling period, no fish were detected. On the other hand, habitat losses as a result of pollution and/or hydraulic structures affected 7 stations negatively (St. 7, 11, 16, 17, 18, 19, and 20) (Table 1). Fish only sampled from 11 stations; six of them were sampled in both seasons (St. 8, 22, 23, 28, 31, and 36) and five stations (St. 15, 21, 25, 33, and 39) were dry in autumn 2017, but there were water and fish in spring 2018. Since the river basin is located in the Mediterranean climate zone, the drought is expected in the riverbed in the summer period with decreasing rainfall. However, the high number of dry stations in the streams throughout the year is remarkable. This situation is not only a result of the meteorological situation but also the dams built for domestic uses or irrigation purposes. It is recommended to release the water to the riverbeds from the dams to keep the ecological processes to continue healthily.

There is a water pollution problem throughout Küçük Menderes River Basin, from the headwater to the Pamucak coast where it flows into the sea. According to field observations, the problem becomes more distinct especially in the settlement areas along the main river branch. The most prominent example of water pollution was observed in two stations located on Fetrek Creek. The first station located before the settlement area (St. 15) was dry, however, the second station was wet with domestic or industrial wastewater flow along the riverbed after the settlement area (St. 16). In order to protect the whole ecosystem, it is necessary to prevent domestic and industrial wastewater discharged to the environment without suitable treatment.

As a result of the fishing done in Küçük Menderes River Basin, 21 fish species were detected (Table 2) and three of them, G. holbrooki, C. gibelio and $C$. 
auratus are invasive-alien fish for the freshwater fish fauna of Turkey (İnnal \& Erk'akan, 2006; Tarkan et al., 2015). Gambusia holbrooki was captured from six stations (Table 2), one of them is the Hursiz Stream, which is the outlet water of the Alaçatı Dam Lake, with high catch efficiency and the other is Kuskudan Stream-1 station, which is the outlet water of Mordoğan Pond (Table 3). In these two stations, the streams are very shallow and have a very weak flow, which is almost stagnant. Although $G$. holbrooki has a wide habitat tolerance, it prefers to be in stagnant or slow-flowing waters compared to fast-flowing waters (García-Berthou, 1999; Pyke, 2005). Since there is no other fish to compete, G. holbrooki, an opportunistic species, is considered to have high catch efficiency at these two stations. The other four stations, where this invasive fish was determined, are the shores of the lentic water bodies (Table 2). They were obtained by using a scoop-net. The catch efficiency at these stations could not be evaluated as no specimen was caught with the standard fishing nets used in the lakes. The other two invasive-alien fish, $C$. gibelio and $C$. auratus, have entered into Turkish inland waters in the early 1980s and their first records were given from the Gala Lake in Evros River Basin (Baran \& Ongan, 1988). Over time, they were introduced to other water basins in Turkey by stocking purposes. C. auratus was detected only from Alaçatı Dam Lake and C. gibelio was detected from one stream station (St. 15, Fetrek Creek) and four lentic water bodies (Table 2). It is quite important to control these three invasive species in the basin and to determine their interactions with native species which many of them are endemic fish species.

Four species, A. boyeri, S. glanis, P. fluviatilis, and C. carpio, which are native members of the freshwater fish fauna of Turkey, are translocated fish for the basin. The most important factor in their voluntary/accidental translocation is fish stocking. They can easily become dominant fish with the absence of natural predators in the inland water environment. They enter or/with their biological features such as reproduction and feeding. These translocated species, which are mostly preferred for economic reasons, may threat to biodiversity (McKinney, 2001; De Silva et al., 2009). It should be preferred to give priority to native fish species while stocking fish in river basins. Also, maximum attention should be shown not to move the unwanted species to the new environment together with the species preferred in fisheries. When an alien fish species enters a new environment, it will be both very difficult and quite expensive to remove it from there.

There are some hydraulic structures such as dams, culverts, flood protection structures, weirs and bridges in Küçük Menderes River Basin that destroy the stability of the river both horizontally and vertically. Besides, the other threats such as to take the natural riverbed in a channel, the destruction of the meanders or riparian 
areas, the fortification of the river banks with concrete or rocks are also remarkable for the basin. All these interventions affect the biodiversity of both the river and the terrestrial area where it interacts. Excluding the five dam lakes, one or more of the hydraulic structures listed above are present in almost all stations. Evaluating the native fish species of Küçük Menderes River basin, different fish species may show different habitat preferences from upstream to downstream; some (A. demiri, $B$. pergamonensis) prefer fast-flowing regions, while others (R. amarus, $P$. smyrnaeus) prefer slow-flowing regions with abundant vegetation; some species ( $C$. fahireae) prefer sandy-muddy ground while others (O. germencicus, $R$. amarus) prefer stony sediment. However, structures in the habitat of these fish are very important threat to the survival of them. Barbus pergamonensis, O. germencicus, P. smyrnaeus and $S$. kosswigi are the endemic fishes living in these streams and further intervention in the riverbed may threaten the existence of these fishes in the future. Especially, in the upstream direction of the Fetrek Creek, there are deep oxbow lakes formed by the closure of the meanders and these small oxbow lakes are thought to have been used as shelters for these endemic fishes as well as other aquatic organisms during arid periods. Therefore, these deep oxbow lakes should be protected, intervention to their natural structure should be avoided and its water should not be used for irrigation. To ensure the stability of the river basin, the coordinates, types and features of all hydraulic structures should be documented and the unnecessary structures should be demolished and environmentally friendly solutions such as fish passages should be developed.

As a result of urbanization, Tahtalı, Beydağ, Kavakdere, Seferihisar, and Alaçatı dams were built for both domestic use and irrigation purposes to meet the needs of the growing population. Although these dams have already pressure on the habitat use of the native fish fauna, the introduced/translocated fishes are also important threats in the dams and the streams of the sub-basins. The Tahtali Dam, built on the Gümüldür Stream in the 1990s, is the most important drinking water body in Izmir city and the streams and their environments in the dam sub-basin are protected by the local authorities. Despite, this sub-basin is rich in endemic species (A. demiri, B. pergamonensis, C. fahireae, O. germencicus, $P$. smyrnaeus and $S$. kosswigi), it is also under threat of invasive ( $C$. gibelio) and translocated (C. carpio and $P$. fluviatilis) fish species. In the Şaşal and Tahtali creeks, the main sources of the Tahtalı Dam, all the fish species are Anatolian endemics except $C$. gibelio (in the Şaşal Creek) and the abundance of these endemics is higher than invasive one, since C. gibelio mostly prefers lentic systems to lotics ones (Table 3). Although it has the potential to become one of the dominant fish of the Tahtali Dam with its reproductive success, it has to share the same biotope with the predators $P$. fluviatilis and S. glanis, 
which were introduced to the dam for stocking purposes. These predator fishes may also control the overgrowth of $C$. gibelio population.

Previous studies reported 19 fish species living in Küçük Menderes River Basin (Karaman, 1972; Balık, 1979; Balık et al., 1995; Sarsu, 1981; İlhan et al., 2009; Korkmaz et al., 2015). Four fish species C. holmwoodii, A. bipunctatus, C. carassius and $M$. cephalus, which are reported from Gümüldür Stream, were not found in the present study. During the sampling period, the lower part of Gümüldür Stream, located at the downstream of the Tahtalı Dam embankment, was dry and seawater had entered to some part of the stream. On the other hand, the fish may have become stuck in a narrow area under changing habitat conditions after the dam construction in the upstream.

Atherina boyeri, C. auratus, S. glanis, S. abaster and K. caucasica were reported from the basin for the first time. It is thought that $A$. boyeri (only in Kavakdere Dam Lake), $C$. auratus and $S$. glanis entered into the basin through fish stocking as mentioned above. Syngnathus abaster are originally marine fish, and the only area which is expected to catch of this fish was Gebekirse Lake because of its connection to the sea. Additionally, K. caucasica, a small-bodied fish that is very difficult to catch with gillnets, captured from two lakes, Kavakdere Dam Lake and Gebekirse Lake, using a small scoop-net indicating the importance of using different fishing gear to reveal the fish fauna in the ecosystems.

In conclusion, the fish fauna of Küçük Menderes River Basin (İzmir) and their abundances have been studied in detail for the first time. Total 21 fish species have been obtained, however, the number of fish species is likely to be higher. The reasons of the low number of fish species could be as follows; only two sampling periods (spring and autumn), pollution and drought in several stations, the limited (1 or 2) number of the stations in the river tributaries and the gillnet/seine net selectivity in lakes, dams and transitional waters. Six fish species (A. boyeri, S. abaster, $C$. aurata, C. ramada, Chelon sp. and $S$. fluviatilis) in the basin are of marine origin with high salinity tolerance and can live in inland waters. Silurus glanis and $P$. fluviatilis are native members of the freshwater fish fauna of Turkey and they were introduced into Küçük Menderes River basin for stocking purposes and these two species may threaten the existence of native fishes by their predation pressures. Therefore, it is very critical to monitor and also determine the consequent effects of these invasive and introduced species on the fish fauna, especially native species, of the basin. In addition, all stakeholders should use water with an ecosystem-based approach and prefer nature-friendly methods to eliminate the threats mentioned above in detail. 


\section{Acknowledgement}

This study was supported by the Ministry of Agriculture and Forestry, General Directorate of Water Management (Project on Management Plan of Küçük Menderes River Basin). 


\section{References}

Balık, S. (1979). Batı Anadolu tatlısu balıklarının taksonomisi ve ekolojik özellikleri üzerine araştırmalar (Publication No. 236) [Doctoral dissertation, Ege University]. Ege Üniversitesi Fen Fakültesi İlmi Raporlar Serisi(in Turkish).

Balık, S., Ustaoğlu, M. R., Sarı, H. M., \& Aygen, C. (1995). Tahtalı Baraj Havzasının (Gümüldürİzmir) Omurgalı Faunası. II. Ulusal Ekoloji ve Çevre Kongresi (pp.463-472). Ankara, Turkey.

Baran, İ. \& Ongan, T. (1988). Gala Gölü’nün Limnolojik Özellikleri, Balıkçılık Sorunları ve Öneriler. Gala Gölü ve Sorunları Sempozyumu, Doğal Hayatı Koruma Derneği Bilimsel Yayınlar Serisi (pp.46-54). (in Turkish)

CEN (European Committee for Standardization) (2003). European Standard EN 14011: Water quality - sampling of fish with electricity.

CEN (European Committee for Standardization) (2006). European Standard EN 14962: Water quality - guidance on the scope and selection of fish sampling methods.

CEN (European Committee for Standardization) (2015). European Standard EN 14757: Water quality - sampling of fish with multi-mesh gillnets.

De Silva, S. S., Nguyen, T. T. T., Turchini, G. M., Amarasinghe, U. S., \& Abery, N.W. (2009). Alien species in aquaculture and biodiversity: a paradox in food production. Ambio: A Journal of the Human Environment, 38(1), 24-28. https://doi.org/10.1579/0044-7447-38.1.24

García-Berthou, E. (1999). Food of introduced mosquitofish: ontogenetic diet shift and prey selection. Journal of Fish Biology, 55, 135-147. https://doi.org/10.1111/j.1095-8649.1999.tb00663.x

Hassan Rashid, M.A.U., Manzoor, M.M. \& Mukhtar, S. (2018). Urbanization and its effects on water resources: An exploratory analysis. Asian Journal of Water, Environment and Pollution, 15(1), 67-74. https://doi.org/10.3233/AJW-180007

Işık, Ş. (2005). Urbanisation and Urbanisation Models in Turkey. Aegean Geographical Journal, 14(1-2), 57-71. (in Turkish with an abstract in English)

İlhan, A., Ustaoğlu, M. R., Sarı, H. M., Başıaçık, S., \& Gürleyen, N. (2009). The reproduction features of perch (Perca fluviatilis L., 1758) population in Tahtalı Dam Lake (Izmir). Ege University Journal of Fisheries and Aquatic Sciences, 26(4), 257-260. (in Turkish with an abstract in English)

İnnal, D., \& Erk'akan, F. (2006). Effects of exotic and translocated fish species in the inland waters of Turkey. Reviews in Fish Biology and Fisheries, 16, 39-50. https://doi.org/10.1007/s11160006-9005-y

Jordan, G.R. \& Willis, D.W. (2001). Seasonal variation in sampling indices for Shovelnose Sturgeon, River Carpsucker, and shorthead redhorse collected from the Missouri River below Fort Randall Dam, South Dakota. Journal of Freshwater Ecology, 16(3), 331-340. https://doi.org/10.1080/02705060.2001.9663820 
Karaman, M.S. (1972). Süßwasserfische der Türkei. 9. Teil. Revision einiger kleinwüchsiger Cyprinidengattungen Phoxinellus, Leucaspius, Acanthobrama usw. aus Südeuropa, Kleinasien, Vorder-Asien und Nordafrika. Mitteilungen aus dem Hamburgischen Zoologischen Museum und Institut, 69, 115-155.

Korkmaz, M., Mangıt, F., \& Yerli, S.V. (2015). Length-weight relationships for freshwater fish species in Küçük Menderes River Basin, Turkey. Frontier in Marine Science Conference Abstract: XV European Congress of Ichthyology. https://doi.org/10.3389/conf.FMARS.2015.03.00167

Kurucu, Y., \& Chiristina, N. K. (2008). Monitoring the impacts of urbanization and industrialization on the agricultural land and environment of the Torbali, Izmir region, Turkey. Environmental Monitoring and Assessment, 136, 289-297. https://doi.org/10.1007/s10661-007-9684-4

McKinney, M. L. (2001). Effect of human population, areas and time on non-native plant and fish diversity in the United States. Biological Conservation, 100, 243-252. https://doi.org/10.1016/S0006-3207(01)00027-1

Mehner, T., Diekmann, M., Brämick, U. \& Lemcke, R. (2005). Composition of fish communities in German lakes as related to lake morphology, trophic state, shore structure and human-use intensity. Freshwater Biology, 50, 70-85. https://doi.org/10.1111/j.1365-2427.2004.01294.x

Özuluğ, M. \& Saç, G. (2019). The freshwater fish fauna of İstanbul province (Turkey). Turkish Journal of Bioscience and Collections, 3(1), 19-36. (in Turkish with an abstract in English) https://dergipark.org.tr/tr/download/article-file/668606

Pompeu, P. S., \& Alves, C. B. M. (2005). The effects of urbanization on biodiversity and water quality in the Rio das Velhas Basin, Brazil. American Fisheries Society Symposium, 47, 11-22.

Pyke, G. H. (2005). A review of the biology of Gambusia affinis and G. holbrooki. Reviews in Fish Biology and Fisheries, 15, 339-365. https://doi.org/10.1007/s11160-006-6394-x

Sarsu, M. (1981). Gümüldür Deresi ve ona bağlı kaynaklarda yaşayan tatlısu balıklarının taksonomisi ve ekolojik özellikleri üzerine ön çalışmalar [Diploma thesis, Ege University]. (in Turkish)

Shukla, S., Khire, M. V., \& Gedam, S. S. (2013). Effects of increasing urbanization on river basinsstate of art. International Journal of Engineering Research \& Technology, 2(12), 2742-2747. https://www.ijert.org/research/effects-of-increasing-urbanization-on-river-basins-state-ofart-IJERTV2IS121108.pdf

Sökmen Y1lmaz, E. \& Y1lmaz, S. (2019). A review on urbanization, pollution and biodiversity in İzmir. International Journal of Environmental Trends, 3(1), 31-38. https://dergipark.org.tr/tr/download/article-file/741836

Sütgibi, S. (2009). Delta ecosystems and degradational impacts: A case study on the Küçük Menderes Delta. Aegean Geographical Journal, 18(1-2), 59-72. (in Turkish with an abstract in English) https://dergipark.org.tr/en/download/article-file/56915 
Tarkan, A. S., Marr, S. M., \& Ekmekçi, F.G. (2015). Non-native and translocated freshwater fish species in Turkey. FiSHMED: Fishes in Mediterranean Environments, 2015.003, 28. http://sibic.org/wp-content/uploads/2015/12/2015.003_Tarkan_etal.pdf

Turkey Statistical Institute. (2020). https://www.tuik.gov.tr

Yıldız, S., Ustaoğlu, M. R., \& Balık, S. (2009). Oligochaeta (Annelida) fauna of Akgöl and Gebekirse Lakes (Selçuk-İzmir). Review of Hydrobiology, 2, 173-186. 


\section{Extended Turkish Abstract (Genişletilmiş Türkçe Özet)}

\section{Kentleşmenin Kü̧̈ük Menderes Nehir Havzası (Türkiye) Balık Toplulukları Üzerindeki Baskısı}

Nehir havzaları, suyolları olmaları nedeniyle biyosferde önemli bir role sahiptirler ve insanlar tarafından ulaşımda, balıkçılıkta, hidroelektrik üretimi ile evsel, endüstriyel ve tarımsal su temini amaçlarıyla kullanılmaktadırlar. Batı Anadolu'nun önemli akarsularından biri olan Küçük Menderes Nehri, Türkiye'nin metropollerinden biri olan İzmir ili boyunca devam ederek Ege Denizi'ne akmaktadır. Kentleşme, balıkları da kapsayan sucul fauna ve florada meydana gelen değişimlerle bağlantılı olup sıklıkla balık çeşitliliği, zenginliği ve yoğunluğunda azalmaya yol açmaktadır. Günümüzde Küçük Menderes Nehri, verimli tarım arazileri ve yüksek sanayi ve turizm potansiyeli nedeniyle yapılaşmanın baskısı altındadır. Bununla birlikte, havzadaki altyapı eksikliği (kanalizasyon, arıtma tesisleri, vb.) ve hızlı nüfus artışı su kirliliğine/kaybına ve su dengesinin bozulmasına neden olmaktadır. Bu çalışmanın amacı, kentleşme baskısı altındaki Küçük Menderes Nehir Havzası'nın balık faunası ile bunların bolluklarını belirlemektir.

Saha çalışmaları, Küçük Menderes Nehir Havzası'ndaki toplam 50 örnekleme istasyonunda (42 akarsu, 1 geçiş suyu, 2 doğal göl ve 5 baraj gölü) Kasım (2017) ve Mayıs (2018) aylarında gerçekleştirilmiştir. Balık örnekleri akarsularda elektroşoker kullanılarak, geçiş sularında ise kıyı sürütme ağıyla toplanmıştır. Göllerdeki balık örneklemeleri çoklu göz açıklıklarına sahip standart bentik ve pelajik galsama ağları ile yapılmıştır. Toplanan örnekler anestezik çözelti ile bayıltıldıktan sonra \%5'lik formaldehit çözeltisine aktarılmıştır. Laboratuvar çalışmasında, her balık için standart boy $(\mathrm{SL}, \mathrm{cm})$ ve toplam vücut ağırlı̆̆ $(\mathrm{W}, \mathrm{g})$ ölçümleri yapılmıştır. Her istasyon için balık bolluğu, birim çaba başına düşen av miktarı (CPUE) hesaplanmıştır.

Çalışılan 50 istasyonun \%38'inde (19 istasyon) balık bulunmasına rağmen diğer istasyonlarda kirlilik (7 istasyon) ve kuruma (24 istasyon) nedeniyle balık yakalanamamıştır. Çalışma sonucunda 14 familyaya ait toplam 21 balık türü elde edilmiştir: Alburnus demiri, Anguilla anguilla, Atherina boyeri, Barbus pergamonensis, Carassius auratus, Carassius gibelio, Chelon ramada, Cobitis fahireae, Cyprinus carpio, Gambusia holbrooki, Knipowitschia caucasica, Chelon aurata, Chelon sp., Oxynoemacheilus germencicus, Perca fluviatilis, Petroleuciscus smyrnaeus, Rhodeus amarus, Salaria fluviatilis, Silurus glanis, Squalius kosswigi ve Syngnathus abaster. Cyprinidae, dört taksonla en yüksek tür zenginliğine sahiptir ve en yaygın olarak bulunan balık türleri $S$. kosswigi (8 istasyon) ve C. carpio (7 istasyon)'dur.

Yakalanan balık türleri bollukları açısından değerlendirildiğinde, akarsu istasyonlarında CPUE (adet $/ \mathrm{dk} / \mathrm{m}^{2}$ ) değerleri 0,01 ile 24,00 arasında değişmiş olup G. holbrooki Hırsız (CPUE=23,28 adet $\left./ \mathrm{dk} / \mathrm{m}^{2}\right)$ ve Kuskudan $\left(\mathrm{CPUE}=24.00 \mathrm{adet} / \mathrm{dk} / \mathrm{m}^{2}\right)$ derelerindeki en baskın balık türüdür. Göl istasyonlarındaki balıklara ait CPUE (adet $/ \mathrm{s} / \mathrm{m}^{2}$ ) değerleri 0,01 ile 62,43 arasında değişmiş olup $P$. fluviatilis Beydağ Baraj Gölü'nde en baskın balık türüdür (CPUE $\left.=62,43 \mathrm{adet} / \mathrm{s} / \mathrm{m}^{2}\right)$. Alburnus demiri, $O$. germencicus, $P$. smyrnaeus ve $S$. kosswigi havzadaki Anadolu endemikleri iken A. boyeri Kavakdere Baraj Gölü için yabanc1, G. holbrooki ise Küçük Menderes Nehir Havzası için yabancı / istilacı balık türüdür.

$\mathrm{Bu}$ çalışmada, sahada yapılan gözlemler sonucunda, temel dört sorun tespit edilmiştir; (1) meteorolojik koşullar ve/veya evsel/endüstriyel kullanımlar ile /tarımsal sulama gibi nedenlerden 
kaynaklı kuruma, (2) su kirliliği, (3) istilacı, yabancı veya taşınmış balıkların varlığı ve (4) barajlar, taşkın koruma yapıları ve menfezler gibi hidrolik yapılardan dolayı nehir sürekliliğinin bozulması. Bazı istasyonlar için ana sorun kuruma olsa da, bazılarında iki veya daha fazla sorunun birlikte etkili olduğu gözlemlenmiştir.

Nehir ekosistemlerinde her iki örnekleme döneminde toplam 31 istasyonda balık yakalanamamıştır. Bunlardan 19'u her iki mevsimde de kuruyken, 5'inde bir örnekleme döneminde su olmasına rağmen balık tespit edilememiștir. Diğer yandan, kirlilik ve/veya hidrolik yapıların etkisi sonucu olarak yaşanan habitat kayıpları 7 istasyonu $(7,11,16,17,18,19$ ve 20) olumsuz etkilemiştir. Balık sadece 11 istasyondan yakalanabilmiştir ve bunların altısında $(8,22,23,28,31$ ve 36$)$ her iki mevsimde su bulunurken beşinde $(15,21,25,33$ ve 39) sadece ilkbahar örneklemesinde su bulunmaktaydı. Akarsulardaki bu kurumanın sadece meteorolojik nedenlerden kaynaklanmadığı, aynı zamanda evsel veya sulama amaçlı inşa edilen barajların etkisiyle de oluştuğu öngörülmüştür.

Küçük Menderes Nehir Havzası boyunca, nehrin membasından denize aktığı Pamucak sahiline kadar su kirliliği sorunu bulunmaktadır. Saha gözlemlerine göre sorun, özellikle ana nehir boyunca uzanan yerleşim alanlarında daha belirgin hale gelmektedir. Su kirliliğinin en belirgin örneği Fetrek Çayı'nda bulunan iki istasyonda gözlenmiştir. Yerleşim alanı öncesinde bulunan ilk istasyon (15. istasyon) kuru iken, yerleşim alanından sonra gelen ikinci istasyondaki akış halindeki suyun tamamen (16. istasyon) evsel veya endüstriyel kökenli olduğu görülmüştür.

Küçük Menderes Nehir Havzası'nda tespit edilen üç balık türü $G$. holbrooki, C. gibelio ve $C$. auratus, Türkiye içsu balık faunası için yabancı ve istilacı özellikteki balıklardır. Bunlara ek olarak tespit edilen diğer dört tür A. boyeri, S. glanis, $P$. fluviatilis ve C. carpio havzaya taşınmış balıklar olarak dikkat çekmektedir. Balık stoklaması çalışmalarında istemli veya istem dışı taşınmalar en önemli faktörlerdir ve bu yabancı balıklar girdikleri iç su ortamında doğal yırtıcıların bulunmaması ya da üreme ve beslenme gibi biyolojik özellikleri ile kolayca baskın balık haline gelebilirler. Çoğunlukla ekonomik nedenlerle tercih edilen bu türler biyolojik çeşitlilik için bir tehdit oluşturabilir.

Küçük Menderes Nehir Havzasında baraj, menfez, taşkın kontrol yapıları, savaklar ve köprüler gibi nehrin hem yatay hem de dikey yöndeki devamlılığını bozan bazı hidrolik yapılar bulunmaktadır. Buna ek olarak, doğal nehir yatağını beton kanala almak, kıyı bölgelerinin tahrip edilmesi, nehir kıyılarının beton veya kayalarla güçlendirilmesi gibi diğer tehditler de havza için dikkat çekicidir. Beş baraj gölü hariç, yukarıda listelenen hidrolik yapıların biri veya daha fazlası hemen hemen tüm istasyonlarda tespit edilmiştir. Barbus pergamonensis, O. germencicus, $P$. smyrnaeus ve $S$. kosswigi bu akarsularda yaşayan endemik balıklardır ve nehir yatağına yapılacak daha fazla müdahalenin gelecekte bu balıkların varlığını tehdit edebileceği düşünülmektedir.

Sonuç olarak, Küçük Menderes Havzası'nın (İzmir) balık faunası ve bollukları bu çalışma ile ilk kez ayrıntılı olarak incelenmiştir. Toplam 21 balık türü elde edilmiştir, ancak tür sayısının daha yüksek olması mümkündür. İstilacı ve taşınmış türlerin havzadaki balık faunası ve özellikle de doğal türler üzerindeki etkilerinin izlenmesi ve belirlenmesi oldukça önemlidir. Ayrıca, tüm paydaşlar ekosistem temelli bir yaklaşımla suyu kullanmalı ve yukarıda belirtilen tehditleri ayrıntılı olarak ortadan kaldırmak için doğa dostu yöntemleri tercih etmelidirler. 